\title{
PHOTOGRAPHY IN SCIENTIFIC RESEARCH
}

A conference was held in Manchester during July 3-4, under the auspices of the Manchester and District Branch of the Institute of Physics, and under the chairmanship of Dr. F. C. Toy, and was devoted solely to photography, with the principal object of giving an opportunity for discussion of the many photographic problems met with in scientific research, where photography plays so large a part. A large amount of time was necessarily given to reviews on the various aspects of photography and its applications, but in the first session fundamental problems of latent image formation were discussed and some original work was reported; this will occupy most of the space available for the present report.

Prof. N. F. Mott opened the session by a "Description of the Photographic Processes in Terms of Atomic Physics", Dr. J. L. M. Brentano, Dr. W. F. Berg and others contributing to the discussion. Dr. S. O. Rawling gave a paper on "Some Theories of the Action of Developers". Much of the matter dealt with has already been reported in NATURE ${ }^{1}$ and elsewhere. The latent image which can so far be revealed only by the process of development was assumed to be a small speck of silver. Modern atomic theory enables a more detailed model of the flocking together of the isolated silver atoms formed by light to be given $^{2}$. The silver halides are photo-conductors, that is, mobile electrons are formed if light is absorbed. The silver halides are also electrolytic conductors: some silver ions (in 'interstitial' positions) will move through the crystal if an electric field is applied. Thus, if there is a place in the crystal where electrons can be collected and held 'trapped', a field will be set up and the ions will move up and neutralize the electrons, thus forming metallic silver. A piece of silver in contact with silver halide constitutes such an electron trap. In this way, once a small speck of silver is formed it will continue to grow if light is absorbed by the crystal. During that process the halogen atoms must escape, otherwise a regression would occur. It is important that in the model suggested the halogen will escape through the crystal surface and will not be liberated at the interface of silver and silver halide. The depth of the electron traps can be determined optically by the Herschel effect: red light will release electrons from the traps and thus bleach the latent image. Thermal energy would not be enough to release electrons, and thus a silver speck in contact with silver halide is stable.
The migration of atoms through solid bodies by a process in which electrons and ions move separately, is a fairly general phenomenon. It occurs, for example, in the atmospheric oxidation of metals, as is shown by the dependence of the rate of that process upon the oxygen pressure ${ }^{3}$. A similar mechanism is responsible for the thermal disintegration of certain azide compounds at $100^{\circ} \mathrm{C} .4$.

To understand the formation of the photographic latent image itself it is necessary to assume the existence of an electron trap before exposure. This probably consists of impurities like silver sulphide, the so-called sensitivity specks ${ }^{5}$. A very small silver speck is considered to be not as stable as a larger one ; electrons and ions may 'evaporate' from, and thus break up, a small speck ${ }^{6}$. This process was regarded as responsible for the 'reciprocity failure' at low intensities; at constant exposure (intensity $\times$ time) the density depends on the intensity level, because at low intensities a speck may partly evaporate, and thus be too small to make the grain developable, whereas at higher intensity the speck reaches a stable size more quickly without losing matter by evaporation.

Some of the experiments reported by Dr. Brentano appeared to require at least some qualification of this picture. These experiments were made on diluted emulsions, thus avoiding the complications due to the multi-layer nature of commercial materials. The shape of the foot of experimental characteristic curves required the assumption that a grain must absorb 10 or more quanta to be made developable. This is of importance for the consideration of low intensity reciprocity failure and suggested experiments in which a certain exposure was given in a few instalments, with varying intervals of time in between. Below and near the threshold, exposures were additive, even when separated by intervals up to 300 hours. Thus, a markedly stable result is obtained already at the very first stages of an exposure. At higher densities, an interruption of an exposure causes a regression, which is greatest where the characteristic curve is steepest. For small exposures an interruption after $\frac{1}{3}, \frac{1}{2}$ or $\frac{2}{3}$ of the exposure has been given causes the same amount of regression. Several breaks even when close together cause a larger regression than a single break. These findings might be accounted for in two ways. One is to assume that the last electron or ion joining a growing speck is for some time in a less consolidated state. The other is to 
modify the assumption of the small specks breaking up and getting lost by a hypothesis introducing a certain delay in the early stages of latent image formation.

Two groups of experiments were made with the intention of showing whether such a delay could be due to a process of temporary trapping of electrons outside the latent image. Experiments in which exposures to light of different wavelength, balanced to produce the same density for the same time of exposure, were added, showed a small but distinct difference in density if the order of the exposures was reversed; this was interpreted as demonstrating a small amount of trapping ${ }^{93}$. An X-ray examination of the state of crystalline perfection of a number of emulsions showed that in general the more sensitive emulsion grains are subdivided into smaller crystallites as indicated by the broadening of the X-ray lines. Another possible interpretation is the existence of a bigger strain in the more sensitive grains. As a particular instance it was mentioned that sensitizing by mercury vapour produced such a broadening. This may be cause or part-effect of the increase in sensitivity.

Reciprocity failure has been shown to be, at least to some extent, a development effect, the rate of development depending on the intensity of the exposures. This effect has not been explained satisfactorily, requiring a deeper insight into the mechanism of development.

The process of development may be explained by a mechanism similar to that of latent image formation, the developer being regarded as a medium that will hand over electrons to the latent image. Thus, a ficld will be set up, and interstitial silver ions will move through the silver halide. For this process to work, the developer must come into intimate contact with the silver speck. The difference in adsorption between various agents, which might otherwise be suitable as developers, may be regarded as responsible for the differences in their behaviour. Another point of view considers the reduction of the silver to occur in the solution, which soon becomes supersaturated and deposits silver on to the latent image. Since the solubility of silver halides in ordinary developers is low, this is probably not the most common process. Accord. ing to still another view, the silver speck is only incidental to latent image formation, which is considered as being the removal of some of the surface charge of the grains. This charge ordinarily inhibits contact between the developer and the grain, but when it is removed, contact becomes possible.

The Gurney-Mott theory $y^{2}$ has given rise to a series of papers undertaken to test that theory. Application of low temperature seemed the obvious means of doing this, since ionic movement is then slowed down or arrested. The experiments lead to the following views'. At low temperatures free electrons are formed and then trapped, partly in the sensitivity specks, partly in other less stable trapping centres in the grain. The depth of the traps could be investigated by releasing the trapped electrons by red and infra-red light. Ionic movements occur on warming up only and depend solely on the total number of electrons trapped, which in turn depends only on the total number of quanta absorbed. Thus, no reciprocity failure should occur at these temperatures, as has actually been shown ${ }^{10,11}$. These experiments, while compatible with the Gurney-Mott theory, cannot be regarded as proving it, since several additional hypotheses were required for their explanation. The value of the theory as a working hypothesis that had given new stimulus to research into photographic problems was stressed.

Mr. E. R. Davies's paper on "Some Important Properties of Photographic Materials" in which the basis for many applications of photographic methods in scientific research were given, and the advantages and dangers of these methods pointed out, was given in his absence by Dr. Berg' ${ }^{12}$. Mr. C. W. Bradley, Dr. J. G. Wilson, Dr. C. A. Adams, Dr. C. A. Bruck and Prof. W. H. Lang then gave examples of the application of photo. graphic methods to specific problems. The three principal advantages of the photographic method lie in the high amplification factor of about $10^{10}$ : a few quanta striking a grain make the whole grain developable ; in the possibility of integrating weak light over long periods-in astronomy the photographic method enables stars to be detected that are ten times weaker than the weakest detectable by the eye; and finally, in the possibility of recording radiation outside the visual range. Some of these advantages have to be paid for; the granular structure leads to loss of resolution due to the graininess and the scattering of light causing 'image spread'. This, however, may sometimes be useful: stellar magnitudes can be determined by the spread of the pin-point images. Many difficulties arise from penetration and exhaustion effects during development, making photographic photometry a difficult problem.

This is further complicated by reciprocity failure, intermittency, and wave-length effects, so that photometry should be based solely on the matching of densities produced by exposures of the same duration and the same degree of intermittency, to light of the same quality and on the same piece of photographic material. Furthermore, the density should be constant over not too small an area. As a beautiful example of this, was mentioned the method of street lighting photography developed in the G.E.C. laboratories ${ }^{13}$. 
During an evening session, to which a much wider audience had been invited, Dr. D. A. Spencer gave an account of present-day colour photography, illustrated by a cartoon film. This lecture was very much appreciated, and the large attendance showed the widespread interest created. The whole of the conference was very well attended, and the discussion was often very lively, demonstrating the need of informal meetings of this type, in which the aim is partly to bring forward new pieces of research, but partly also to relate already published information of the type not easily accessible to the physicist in industry. The hospitality and general arrangements pro- vided by the Physics Department of the University of Manchester were very much appreciated by all participants. IV. F. BERG.

NATCRE, 140, 997 (1937).

- Gurney and Mott, Proc. Roy. Soc., A, 164, 151 (1938).

'Wagner, Z. phys. Chem., B, 21, 25 (1933).

- Papers by Garner, Wischin and IIott, Proc. Roy. Soc. In the Press.

- Sheppard, Phot. J., 65, 380 (1925).

- Fvans and Webb, J. Opt. Soc. Amer., 28, 431 (1938).

? Webb, Phot. J., 76, 78 (1936).

- Strock, Skrifter utgitt av det Yorsk Videnskaps-Akademi i Oslo, (I) Inat.-Naturv. Klasse, No. 10 (1933).

- Berg, Transi Farad. Soc., 35, 145 (1939).

is ibid., footnote, p. 458 .

${ }^{10}$ Berg and Mendelssohn, Proc. Roy. Soc., A, 163, 163 (1933).

$"$ Evans and Hirschlaff, J. Opt. Soc. Amer., 29, 104 (1939).

${ }^{13}$ Juch of the matter of this lecture may be found in Ross, "The Physies of the Ieveloped Photographic Image" (New York, 1924). " Hopkinson, Phot. J.. 76, 323 (1936).

\section{OBITUARIES}

\section{Prof. J. Mellanby}

$\mathrm{T}$ HE death of Prof. John Nellanby on July 15 at the age of sixty years takes from us not only a great physiologist but also a notably genial and kindly personality. He will long be remembered by his colleagues and his students for his penetrating wisdom as well as for his ever-ready willingness to help.

Mellanby went up to Emmanuel College, Cambridge, in 1896 and took Part II of the Science Tripos in 1900 , having been an early student under Gowland Hopkins, who had just begun to teach biochemistry at Cambridge at that time. From Cambridge he went to the research laboratory newly founded by Burroughs and Welleome at Brockwell Hall, and worked there chiefly on the properties of serum proteins and their relation to antitoxins. He left there to complete his clinical course at Manchester and took his Mr.D. at Cambridge in 1907 and returned to the Cambridge laboratory as George Henry Lewes student. Ho was still engaged on protein solutions, and in particular on the phenomenon of clotting in blood and milk.

In 1909 Mellanby left Cambridge to take charge of the Physiological Department of St. Thomas's Hospital and began to use the measurement of the clottingtime of milk as a sensitive method for determining the quantity of active trypsin in pancreatic juice, and thus to work out the details of the formation of trypsin from trypsinogen. From the properties of the juice he went on to elucidate the mechanism of its secretion, and it is perhaps his work on this and on the purification of secretin which, together with his earlier investigation of clotting, form his best-known contributions to knowledge.

Mellanby became a fellow of the Royal Society in 1929 and at the time of his death was a member of its Council and of the Medical Research Council. But his purely physiological work was only one part of his activity. What was always in his mind was the encouragement of research from the clinical side, and he was at all times anxious to help, both by advice and in practice, in any problem that might be brought to him. Ho felt that problems in medicine were problems in physiology and that both were only to be solved by experiment. Such problems arose in the most varied fields and were responsible. for example, for his work with Anwyl-Davies on the anti-coagulant action of arsenobenzol and the making of colloidal gold solution, and with C. R. Box on glycosuria.

In 1937 Mellanby became professor of physiology at Oxford and continued there the work on visceral movement to the study of which he had been led by his work on the effects of secretin. Ho finished an investigation of the changes in size of the spleen shortly before his death. In Oxford, as in London, he was in close touch with his elinical colleagues and his influence on the future of the Nuffield Foundation would have been of immense value. As a researcher he was outstandingly original and versatile, and it is safo to say that time will enlance his reputation in the fields which he made his own.

\section{J. WOOLLEY.}

\section{Dr. M. A. Usov}

The death occurred on July 26 of one of the most eminent Soviet geologists, Mikhail Antonovich Usov, member of the Academy of Sciences of the U.S.S.R., and director of the All-Union Institute of Coordinated Geological Research.

Usov's work was mainly concerned with questions of tectonics and petrology. Ho elaborated a new method for the study of the tectonics of coal deposits, making use of the vast materials available from underground workings, to which geologists had until then paid little attention. An analysis of the breaks he had observed induced him to make an exhaustive classification of the forms of volcanic dislocations. This resulted in the appearance of several works on the tectonics both of individual mines and of the entire Kuznetsk Basin. In these works he revealed 